\title{
Espumas vítreas produzidas a partir de resíduos sólidos
}

\section{Synthesis and characterization of foam glass from solid waste}

\author{
Eduarda Medran Rangel ${ }^{1}$, Caio Cesar Nogueira de Melo ${ }^{1}$, \\ Caroline de Oliveira Carvalho ${ }^{1}$, Alice Gonçalves Osorio ${ }^{1}$,
} Fernando Machado Machado ${ }^{1}$

\footnotetext{
${ }^{1}$ Laboratório de Pesquisa em Materiais - LAPEM, Centro de Desenvolvimento Tecnológico - CDTec, Universidade Federal de Pelotas - UFPel, R. Gomes Carneiro 1, 96010-610, Pelotas, RS, Brasil.

e-mail: eduardamrangel@gmail.com, caiocnm@gmail.com, carolineoli.car@gmail.com, osorio.alice@gmail.com, fernando.machado@hotmail.com.br
}

\section{RESUMO}

O crescimento populacional aliado ao consumo elevado tem aumentado consideravelmente a geração de resíduos sólidos. Diversos são os impactos negativos gerados pela destinação incorreta desses rejeitos. Logo, a utilização de resíduos é cada vez mais atraente tanto no âmbito econômico quanto no ambiental. Neste sentido, no presente trabalho é demonstrada a produção de espumas vítreas, com baixo impacto ambiental, utilizando como matéria-prima resíduos de vidro de lâmpadas fluorescentes e casca de ovo vermelho como agente espumante. Os agentes precursores tiveram as suas composições químicas, estruturas cristalinas e granulometrias elucidadas através das técnicas de fluorescência de raios X, difração de raios X e análise de dispersão a laser, respectivamente. A análise termogravimétrica foi utilizada para avaliar a perda de massa da casca de ovo vermelha durante o aquecimento. As espumas vítreas foram formuladas com $7 \%$ em massa de agentes espumantes, conformadas por prensagem uniaxial e queimados entre $650{ }^{\circ} \mathrm{C}$ e $850{ }^{\circ} \mathrm{C}$, com taxa de aquecimento de $2,5^{\circ} \mathrm{C} / \mathrm{min}$. A influência da temperatura de queima na expansão, na densidade e na porosidade dos corpos cerâmicos obtidos foi avaliada. As espumas tiveram a suas expansões volumétricas avaliadas, sendo suas microestruturas caracterizadas por microscopia óptica. Os resultados mostraram a produção de espumas vítreas altamente porosas, com alta expansão, entre de 122 a $266 \%$, e com baixa densidade, variando de 0,37 a $0,65 \mathrm{~g} . \mathrm{cm}^{-3}$, sendo que os melhores resultados foram alcançados a $750{ }^{\circ} \mathrm{C}$. O material obtido possui grande potencial para aplicações na indústria civil, como isolante térmico e acústico, além de minimizar os impactos ambientais causados pelo descarte indevido de lâmpadas fluorescentes e dos resíduos da casca de ovos.

Palavras-chave: Resíduos sólidos, temperatura de queima, expansão volumétrica, porosidade, material sustentável.

\section{ABSTRACT}

Population growth coupled with high consumption has considerably increased the generation of solid waste. Several are the negative impacts generated by the incorrect destination of these wastes. Therefore, the use of waste is becoming attractive both in economic and in environmental terms. In this sense, the present work demonstrates the production of foam glass with low environmental impact, using as raw material glass residues of fluorescent lamps and red eggshell as a foaming agent. The precursor agents had their chemical compositions, crystal structures and granulometry elucidated by X-ray fluorescence, X-ray diffraction, and laser scattering analysis techniques, respectively. The thermogravimetric analysis was used to evaluate the loss of mass of the red eggshell during the heating. The foam glass was formulated with $7 \mathrm{wt} \%$ of foaming agents, shaped by uniaxial pressing and burned between $650{ }^{\circ} \mathrm{C}$ and $850{ }^{\circ} \mathrm{C}$ at a heating rate of $2.5^{\circ} \mathrm{C} / \mathrm{min}$. The influence of firing temperature in the expansion, density, and porosity of the ceramic cups obtained was evaluated. The foam glasses have their volumetric expansions evaluated, and their microstructures were characterized by light microscopy. The results showed the production of foam glass highly porous glassy with high expansion between $122-266 \%$, and low-density ranging from 0.37 to $0.65 \mathrm{~g}_{\mathrm{cm}} \mathrm{cm}^{-3}$, and the best results were achieved at $750{ }^{\circ} \mathrm{C}$. The material obtained has great potential for applications in the civil industry, such as thermal and acoustic insulation, as well as the decrease of environmental impacts caused by the undue dis- 
charge of fluorescent lamps and egg shell residues.

Keywords: Solid waste, burning temperature, volumetric expansion, porosity, sustainable material.

\section{INTRODUÇÃO}

A produção de bens de consumo a base de vidro reciclado pode ser considerada um caminho promissor para o tratamento de diversos tipos de resíduos sólidos. De uma forma geral, os vidros possuem uma elevada estabilidade química, baixa condutividade térmica, baixo valor de matéria-prima aliada a facilidade de processamento [1]. Dentre os principais produtos confeccionados utilizando o vidro como matéria-prima, destacam-se as garrafas, as embalagens de alimentos e frascos, os painéis automotivos e residenciais, os monitores de computador e televisores, as lâmpadas, entre outros. Dependendo da composição do vidro utilizado na manufatura desses produtos, existe uma grande dificuldade de reutilização e armazenamento após a sua vida útil $[2,3,4]$. Essa dificuldade é justificada pelo alto teor de metais pesados presentes em algumas dessas composições que, quando expostas a intempéries, podem lixiviar [2,5].

Dentre os produtos à base de vidro, destacam-se as lâmpadas fluorescentes. Essa classe de lâmpadas é extremamente utilizada devido a sua alta eficiência e baixo consumo de energia [6]. Anualmente são consumidas em torno de 100 milhões de lâmpadas fluorescentes no Brasil e boa parte dessas lâmpadas são descartadas de forma incorreta após a sua vida útil [7]. Aproximadamente 94\% das lâmpadas fluorescentes são descartadas em aterros sanitários sem as devidas precauções, o que pode acarretar na contaminação do solo e da água por metais pesados, especialmente pelo mercúrio [7]. Esse metal, que pode permanecer biodisponível por vários anos em diversos ambientes, pode causar múltiplos danos a seres vivos como, por exemplo, distúrbio nos sistemas cardiovascular e nervoso, alterações hormonais e reprodutivas, já constatada em peixes predadores e outros animais selvagens expostos a esse metal [7,8,9].

Diversas são as formas de reciclagem de sucata a base de vidro [10,11]. Além da reutilização desse material no próprio processo produtivo, permitindo uma vantajosa diminuição da energia necessária para sua produção, os resíduos de vidro podem ser utilizados como agregado para cimento Portland e concreto asfáltico [11]. Uma alternativa atraente para reciclagem de vidro é a produção de espuma vítrea (EV) $[10,11,12,13,14]$. Essa é alcançada pelo acréscimo de um agente espumante (AE) ao vidro moído e queimado em temperaturas acima da sua temperatura de sinterização [14,15].

Atualmente, as EVs vêm sendo cada vez mais empregadas como isolantes térmicos e acústicos em construções industriais, estruturas comerciais e residenciais $[12,13,16]$. Os benefícios desse tipo de material para aplicações como isolantes térmicos são características como baixa condutividade térmica, estabilidade dimensional, baixa densidade aparente, resistência estrutural superior e temperaturas de operação mais altas que os materiais isolantes convencionais, como espumas poliméricas orgânicas, fibras de vidro ou fibras de lã mineral $[14,15]$.

Um dos empecilhos para a vasta aplicação de EVs consiste no alto custo de sua produção. Entretanto, com a utilização de matérias-primas mais baratas, pode-se prever um crescimento consistente na aplicação de espumas vítreas como substituto de espumas convencionais, tais como as orgânicas [11]. Uma opção para baratear os custos de produção desses corpos cerâmicos é a utilização de resíduos como $\mathrm{AE}$, como a casca de ovo. Esse resíduo é gerado em grandes quantidades, principalmente na cadeia produtiva da indústria alimentícia [17]. Sua utilização é proposta devida a sua composição química [10]: a casca de ovo in natura é composta de carbonato de cálcio na forma de $\mathrm{CaCO}_{3}(94 \% \mathrm{em}$ massa), fosfato de cálcio ( $1 \% \mathrm{em}$ massa), carbonato de magnésio ( $1 \%$ em massa) e matéria orgânica (4\% em massa) [17]. Portanto, as cascas de ovos podem ser consideradas fontes naturais de $\mathrm{CaCO}_{3}$, material amplamente utilizado como $\mathrm{AE}$ na produção de $\mathrm{EV}$ $[5,10,11]$. Assim, a utilização de casca de ovo pode reduzir significativamente o impacto ambiental causado pela prospecção do $\mathrm{CaCO}_{3}$, além de preservar as reservas de calcário, que representam uma fonte natural não renovável.

No presente trabalho, resíduos de lâmpadas fluorescentes e de cascas de ovos vermelhos foram utilizados como materiais precursores na síntese de espumas vítreas. O material obtido possui grande potencial para aplicações na construção civil como isolante térmico e acústico, além de ser um material sustentável e com baixo impacto ambiental.

\section{MATERIAIS E MÉTODOS}

\subsection{Materiais precursores}

$\mathrm{O}$ vidro de resíduo de lâmpadas fluorescentes utilizado para produção dos corpos de prova $(\mathrm{CP})$ foi preparado 
através de um processo de separação - descontaminação, que consiste em capturar todo o vapor de mercúrio através de um filtro de carvão ativado [18]. Como AE, foram utilizadas cascas de ovos vermelhos, sem a película interna, que foram coletadas e armazenadas em estufa a $50^{\circ} \mathrm{C}$.

\subsection{Caracterização dos materiais precursores}

As composições químicas do resíduo de vidro de lâmpadas fluorescentes e da casca de ovo vermelha foram determinadas através da técnica de fluorescência de raios X em um equipamento Shimatzu, modelo XRF 1800. As distribuições granulométricas do vidro, bem como a do agente espumante, foram avaliadas por granulometria a laser em um granulômetro CILAS, modelo 1180 (equipamento com repetibilidade melhor que $1 \%$ e exatidão $<3 \%$ ). A técnica de difração de raios $\mathrm{X}$ foi utilizada para verificar a estrutura cristalina da casca de ovo e do vidro. Para tanto, foi utilizado um difratômetro de raios X Bruker, modelo D2 PHASER, equipado com ânodo de cobre, operado a $30 \mathrm{kV}$ e $10 \mathrm{~mA}$, com os seguintes parâmetros de ensaio: intervalo angular de varredura $10^{\circ}<2 \theta<80^{\circ}$ com passos de $0,05^{\circ}$, por um período de 1 s para cada passo. Para avaliar a perda de massa da casca de ovo vermelha durante o aquecimento, realizou-se uma análise termogravimétrica com o auxílio de termobalança Harrop STA-726 até $1000{ }^{\circ} \mathrm{C}$ a uma taxa de aquecimento de $10{ }^{\circ} \mathrm{C} / \mathrm{min}$.

\subsection{Produção dos corpos de prova}

Tanto o vidro quanto as cascas de ovos vermelhos foram moídos em um moinho de bolas e posteriormente foram peneirados em uma peneira \#200. Os CPs foram confeccionados utilizando $7 \%$ em massa de casca de ovo vermelho. Esse percentual foi escolhido após vários testes preliminares com diferentes percentuais de casca de ovo vermelho (variando de $1 \%$ até $9 \%$ em massa). A formulação foi homogeneizada e umidificada com água e álcool polivinílico (DINÂMICA). Após, a massa homogeneizada foi granulada em peneira \#20 para ser então conformada. Os CPs foram preparados por prensagem uniaxial (RIBEIRO, modelo RP0003) a $40 \mathrm{MPa}$ utilizando uma matriz de aço com as dimensões $83 \times 12 \mathrm{~mm}^{2}$ e secos ao ar por $24 \mathrm{~h}$ e em estufa a $100{ }^{\circ} \mathrm{C}$ por mais $24 \mathrm{~h}$. Os corpos a verde foram queimados em um forno elétrico nas temperaturas de $650^{\circ} \mathrm{C}$, $700{ }^{\circ} \mathrm{C}, 750{ }^{\circ} \mathrm{C}, 800{ }^{\circ} \mathrm{C}$ e $850{ }^{\circ} \mathrm{C}$, com uma taxa de aquecimento de $2,5^{\circ} \mathrm{C} / \mathrm{min}$ e tempo de patamar de $30 \mathrm{~min}$.

Após a queima, determinou-se a expansão volumétrica pela inserção dos corpos cerâmicos dentro de uma proveta, no interior do volume pré-calculado de um material em pó - alumina eletrofundida na granulometria $<180 \mu \mathrm{m}$ - onde, o acréscimo em altura, devido ao deslocamento do pó, forneceu dados para o cálculo do volume [10,11]. Com a determinação do volume pelo deslocamento de massa, pode-se calcular a densidade $(\rho)$ dos CPs $[10,11]$. As equações (1) e (2) foram utilizadas para os cálculos de expansão volumétrica e $\rho$, respectivamente:

$$
\text { Expansão Volumétrica }(\%)=\left(\frac{V_{f}-V_{i}}{V_{i}}\right) \times 100
$$

$$
\rho=\frac{m}{V}
$$

onde $V_{i}$ é o volume após a secagem $\left(\mathrm{cm}^{3}\right)$ e $V_{f}$ é o volume após a queima $\left(\mathrm{cm}^{3}\right), m$ é a massa após a queima $(\mathrm{g})$ e $V$ é o volume após a queima $\left(\mathrm{cm}^{3}\right)$.

A análise microestrutural dos corpos cerâmicos obtidos foi realizada por microscopia óptica em um microscópio OLYMPUS modelo BX41-M LED, com câmera digital Bioptika CMOS Digital Colorida de 5.0 MP acoplada. Com o auxílio do software de análise de imagens Image J (National Institutes of Health, USA) foram mensurados os tamanhos médios de poros das EVs obtidas. Para tanto, foram analisadas as seções transversais de três espumas vítreas obtidas para cada formulação e em cada seção foram analisadas três regiões distintas.

Todas as experiências foram realizadas em triplicata para assegurar a reprodutibilidade, confiabilidade e precisão dos dados. 


\section{RESULTADOS E DISCUSSÃO}

A tabela 1 apresenta a composição química dos materiais precursores utilizados na produção das EVs. Através dessa, é possível observar que o processo utilizado na descontaminação do resíduo de vidro de lâmpadas fluorescentes foi eficiente, pois não existem traços de $\mathrm{Hg}$, o que permitiu a utilização segura do resíduo na fabricação dos corpos cerâmicos [18]. Ainda, é possível verificar que a sua composição é formada principalmente por compostos a base $\mathrm{Si}, \mathrm{Ca}$ e $\mathrm{Na}$, além de pequenas concentrações de outros elementos.

A casca de ovo constitui-se primariamente de $\mathrm{Ca}(92,3 \%)$ seguido de outros compostos a base de $\mathrm{Mg}$, $\mathrm{S}, \mathrm{P}$ e Sr, conforme já observado na literatura $[19,20]$.

Tabela 1: Composição química do vidro e da casca de ovo vermelho.

\begin{tabular}{c|c|c|c}
\hline $\begin{array}{c}\text { AN } \\
\text { ALITO }\end{array}$ & $\begin{array}{l}\text { RESÍDUO DE LÂM- } \\
\text { PADA } \% \text { FLUORESCENTE }\end{array}$ & ANA & $\begin{array}{c}\text { CASCA DE } \\
\text { VERMELHO (\%) }\end{array}$ \\
\hline $\mathrm{Si}$ & 76,4 & $\mathrm{Ca}$ & 92,3 \\
\hline $\mathrm{Ca}$ & 11,6 & $\mathrm{Mg}$ & 1,5 \\
\hline $\mathrm{Na}$ & 2,5 & $\mathrm{~S}$ & 1,0 \\
\hline $\mathrm{Al}$ & 1,9 & $\mathrm{P}$ & 0,8 \\
\hline $\mathrm{K}$ & 1,9 & $\mathrm{Sr}$ & 1,2 \\
\hline $\mathrm{Pb}$ & 1,1 & $\mathrm{Na}$ & 0,8 \\
\hline $\mathrm{Ba}$ & 1,0 & $\mathrm{Si}$ & 1,1 \\
\hline $\mathrm{Mg}$ & 0,9 & $\mathrm{Cl}$ & 0,3 \\
\hline $\mathrm{P}$ & 0,9 & $\mathrm{Fe}$ & 0,5 \\
\hline $\mathrm{Fe}$ & 0,8 & $\mathrm{~K}$ & 0,2 \\
\hline $\mathrm{S}$ & 0,4 & $\mathrm{Al}$ & 0,2 \\
\hline $\mathrm{Y}$ & 0,3 & & \\
\hline $\mathrm{Sr}$ & 0,2 & & \\
\hline $\mathrm{Zr}$ & 0,1 & & \\
\hline
\end{tabular}

A Figura 1 apresenta os difratogramas de raios $\mathrm{X}$ da casca de ovo e do resíduo de vidro. Por meio do difratograma da Fig. 1(A), é possível observar máximos de difração em aproximadamente $23^{\circ}, 29^{\circ}, 36^{\circ}, 39^{\circ}$, $43^{\circ}, 47^{\circ}$ e $48^{\circ}$, que são característicos do carbonato de cálcio (Calcita, $\mathrm{CaCO}_{3}$, JPDS Card 00-005-0586), com estrutura cristalina do tipo romboédrica. Esse resultado está de acordo com o resultado obtido na análise por fluorescência de raios X, que apresentam o Ca como elemento predominante na composição das cascas de ovo. O difratograma de raios $\mathrm{X}$ da amostra de resíduo de lâmpada fluorescente (Fig. 1(B)) apresenta uma banda larga entre $20^{\circ}<2 \theta<40^{\circ}$, típica de um material amorfo [10].
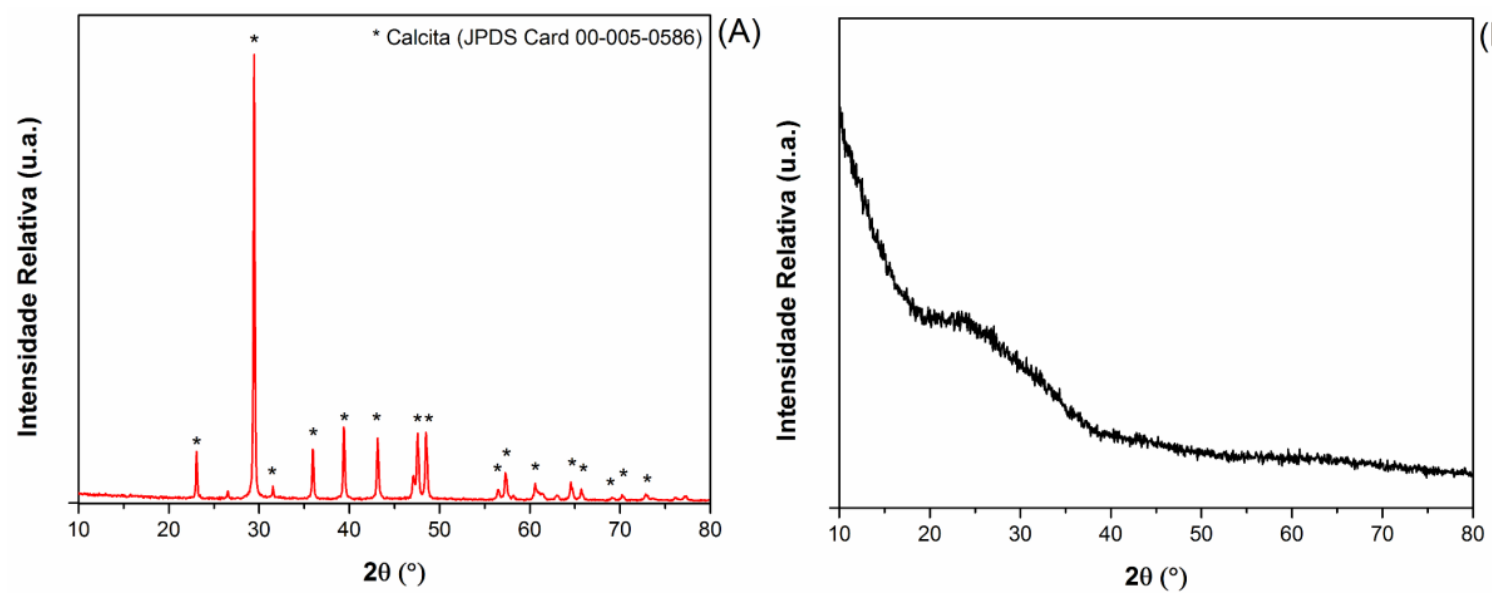

Figura 1: Difratogramas de raios X da (A) casca de ovo vermelha e (B) do resíduo de lâmpada fluorescente. 
A Tabela 2 apresenta os valores da distribuição granulométrica das matérias-primas precursoras das EVs. A influência da granulometria do vidro sodo-cálcico no processo de espumação da espuma vítrea pode ocorrer tanto no fenômeno de aprisionamento - liberação de $\mathrm{CO}_{2}$ oriundo da decomposição do $\mathrm{CaCO}_{3}$ quanto no processo de formação de fase vítrea [21]. Uma partícula maior de vidro apresenta menor reatividade, logo a reação entre a matéria-prima e o agente espumante ocorre mais lentamente, formando uma porosidade não homogênea [22].

König et al. [23] relatam que para a formação de uma EV com baixa densidade, o tamanho máximo da partícula $\left(D_{90}\right)$ de vidro e do $A G$ devem ser inferiores a $163 \mu \mathrm{m}$ e $27 \mu \mathrm{m}$, respectivamente. Os resultados de distribuição granulométrica obtidos nesse trabalho para vidro de lâmpada fluorescente e a casca de ovo apresentam características necessárias para a obtenção de um produto final de boa qualidade, pois os $\mathrm{D}_{90}$ desses materiais são 63,29 e $25,32 \mu \mathrm{m}$, respectivamente.

Tabela 2: Valores da distribuição granulométrica das matérias-primas precursoras.

\begin{tabular}{|c|c|c|c|c|c|}
\hline $\begin{array}{l}\text { MATÉRIA- } \\
\text { PRIMA }\end{array}$ & $(\mu \mathrm{m}) \mathrm{D}_{10}$ & $(\mu \mathrm{m}) \mathrm{D}_{50}$ & $(\mu \mathrm{m}){ }^{D_{90}}$ & $(\mu \mathrm{m})$ & D MÉDIO \\
\hline Vidro de lâmpada \#200 & 5,8 & 29,7 & 63,3 & 32,9 & \\
\hline Casca de ovo vermelho \#200 & 0,8 & 4,2 & 25,3 & 8,9 & \\
\hline
\end{tabular}

A Figura 2 apresenta a análise termogravimétrica da amostra de casca de ovo vermelho. Por meio dessa, pode-se observar a presença de duas zonas de perda de massa: a primeira por volta de $250{ }^{\circ} \mathrm{C}$, referente à perda da água adsorvida, e a segunda entre $650{ }^{\circ} \mathrm{C}$ até $770{ }^{\circ} \mathrm{C}$, referente a decomposição do $\mathrm{CaCO}_{3}[10]$. Para a casca de ovo, acima de $800^{\circ} \mathrm{C}$ restam predominantemente $\mathrm{CaO}$, além de resíduos referentes às cinzas e compostos inorgânicos [24]. A perda de massa total foi de aproximadamente 44,2\%.

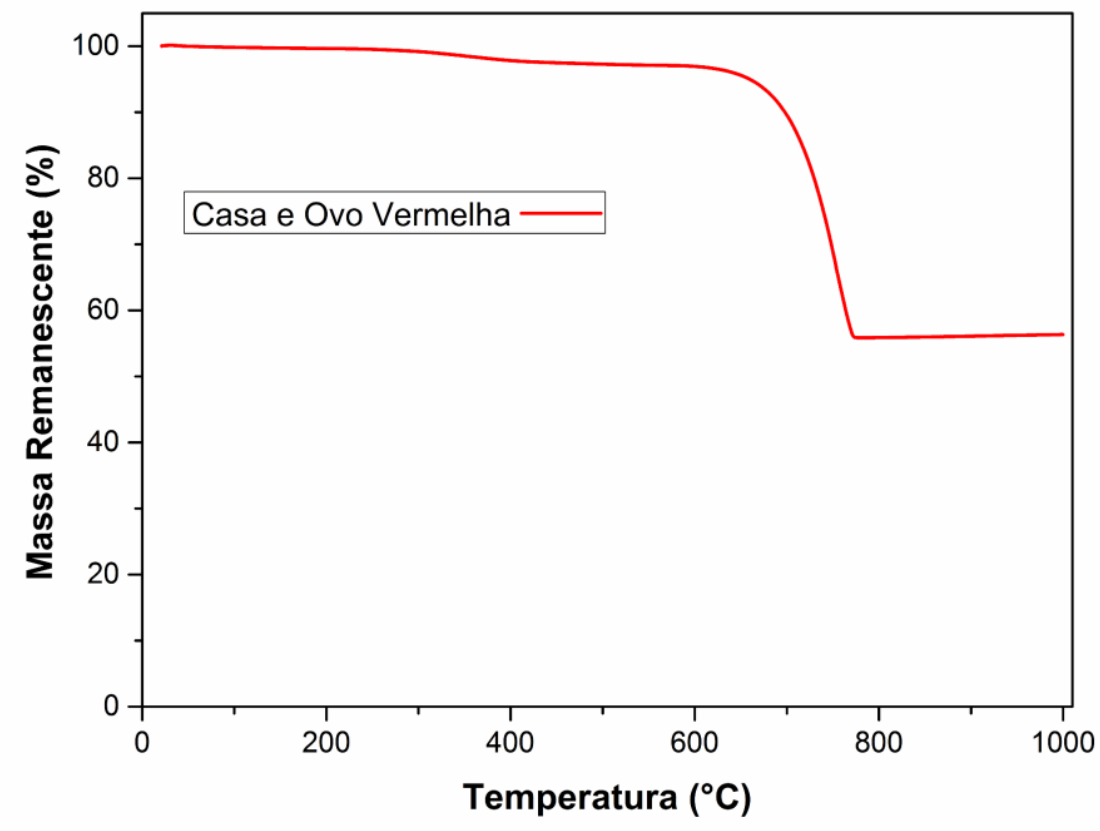

Figura 2: Análise da massa remanescente do agente espumante em função da temperatura. 
A Figura 3 apresenta os resultados da expansão volumétrica das EVs formuladas com $7 \%$ de AE, em função da temperatura de queima. Através dessa figura é possível observar que as AEs começaram a expandir a partir da temperatura de $650{ }^{\circ} \mathrm{C}$. Ainda, a expansão é progressiva até $750{ }^{\circ} \mathrm{C}$, temperatura na qual ocorre a maior taxa de expansão volumétrica, de $266 \%$, e a partir dessa temperatura ocorre o decréscimo da expansão. Para que o processo de formação da EVs seja bem-sucedida é essencial que os gases, formados pela decomposição do $\mathrm{AE}$, sejam libertados dentro matriz vítrea entre as temperaturas de sinterização e amolecimento do vidro precursor $[5,10,23,25]$. Isso garante que os gases não sejam perdidos pela porosidade aberta da peça e ainda, que a viscosidade da fase vítrea seja suficiente para aprisionar as bolhas formadas pela decomposição do AE [10,23]. Através da análise termogravimétrica (Fig. 2), pode-se observar que a decomposição do AE ocorre na faixa de 650 e $770{ }^{\circ} \mathrm{C}$. Quando a casca de ovo tem a sua temperatura elevada acima de $650{ }^{\circ} \mathrm{C}$, essa começa a sofrer decomposição térmica, dando origem a óxido de cálcio e a $\mathrm{CO}_{2}$ [10,21]. A emissão do $\mathrm{CO}_{2}$ dentro do $\mathrm{CP}$ promove a espumação, criando poros e a consequente expansão dos corpos cerâmicos. A melhor eficiência de decomposição térmica - aproveitamento do AE ocorre em aproximadamente $750{ }^{\circ} \mathrm{C}$. Vale ressaltar que essa temperatura está acima da temperatura de sinterização do resíduo de vidro, que é de aproximadamente $685{ }^{\circ} \mathrm{C}$ e abaixo da temperatura de amolecimento, que é de aproximadamente $825{ }^{\circ} \mathrm{C}$ [10]. Em temperaturas superiores a $750{ }^{\circ} \mathrm{C}$, as bolhas formadas a partir da decomposição do AE entram em colapso e escapam da matriz vítrea [21].

Por meio da Figura 4, é possível visualizar que a produção do CP com menor densidade, de aproximadamente $0,37 \mathrm{~g} / \mathrm{cm}^{3}$, ocorreu na temperatura de $750{ }^{\circ} \mathrm{C}$. Esse valor está muito próximo da faixa de densidade de produtos comerciais, que é de aproximadamente $0,3 \mathrm{~g} / \mathrm{cm}^{3}[10,22]$.

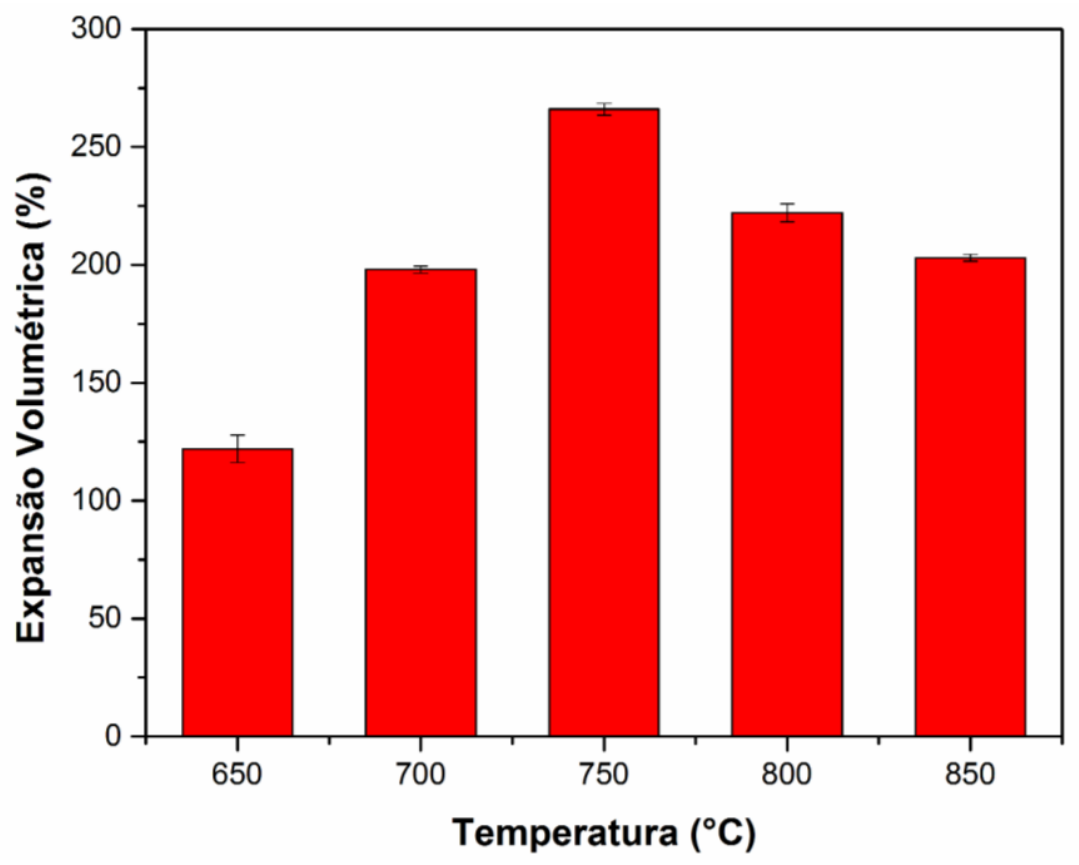

Figura 3: Valores de expansões volumétricas dos CPs obtidos para as diferentes temperaturas de queima. 


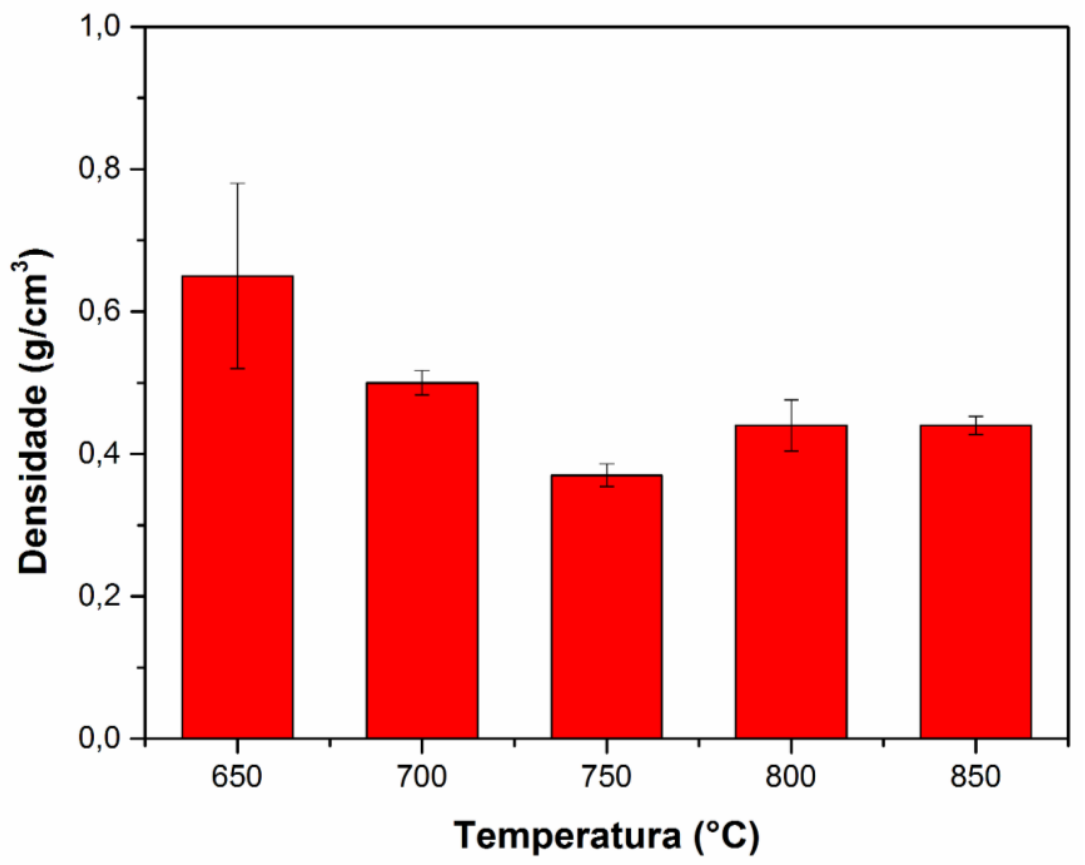

Figura 4: Valores de densidade dos CPs obtidos para as diferentes temperaturas de queima.

A Figura 5 apresenta a aparência da macroestrutura (observada na secção transversal) do CP a verde e também dos CPs obtidos nas diferentes temperaturas de queima. É possível observar que a porosidade está diretamente ligada a temperatura de queima, pois aumentando a temperatura de queima a porosidade fica mais aberta e menos homogênea. Ainda, pode-se verificar que as EVs obtidas a $650^{\circ} \mathrm{C}$ (Fig. 5(B)) não apresentam porosidade aparente devido à baixa decomposição do AE nessa temperatura (ver Fig.2). Já nos CPs queimados nas temperaturas de 700 e $750{ }^{\circ} \mathrm{C}$ (Figs. $5(\mathrm{C})$ e (D)) é possível verificar uma grande quantidade de poros não homogêneos na estrutura fechada. A partir de $800{ }^{\circ} \mathrm{C}$ (Fig. 5 (E)) a porosidade já se apresenta aberta, obtendo-se poros na superfície da amostra.

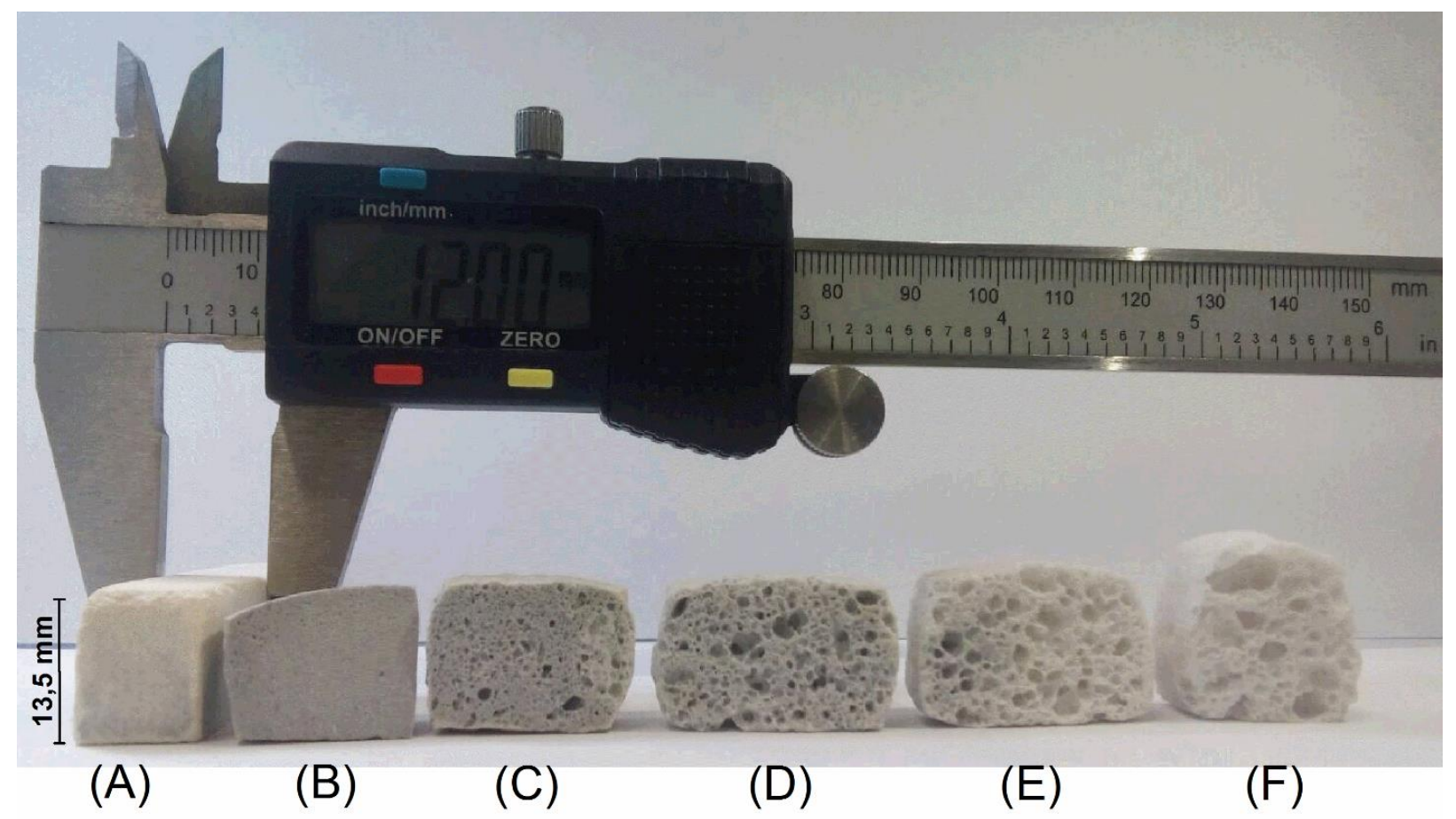

Figura 5: Aparência da macroestrutura observada na secção transversal do (A) corpo a verde e dos CPs obtidos a (B) $650{ }^{\circ} \mathrm{C},(\mathrm{C})$ a $700{ }^{\circ} \mathrm{C},(\mathrm{D})$ a $750{ }^{\circ} \mathrm{C},(\mathrm{E})$ a $800{ }^{\circ} \mathrm{C}$ e $(\mathrm{F})$ a $850{ }^{\circ} \mathrm{C}$. 
A Figura 6 e a Tabela 3 apresentam, respectivamente, as imagens adquiridas através da microscopia óptica e os valores de diâmetro médio de poros (mensurados com o auxílio do software de análise Image J) para as espumas vítreas obtidas nas temperaturas de 700 a $850{ }^{\circ} \mathrm{C}$. A micrografia e valor de diâmetro médio de poros da EV sintetizada a $650{ }^{\circ} \mathrm{C}$ não são apresentados, pois essa não desenvolveu porosidade aparente considerável (ver Fig. 5).
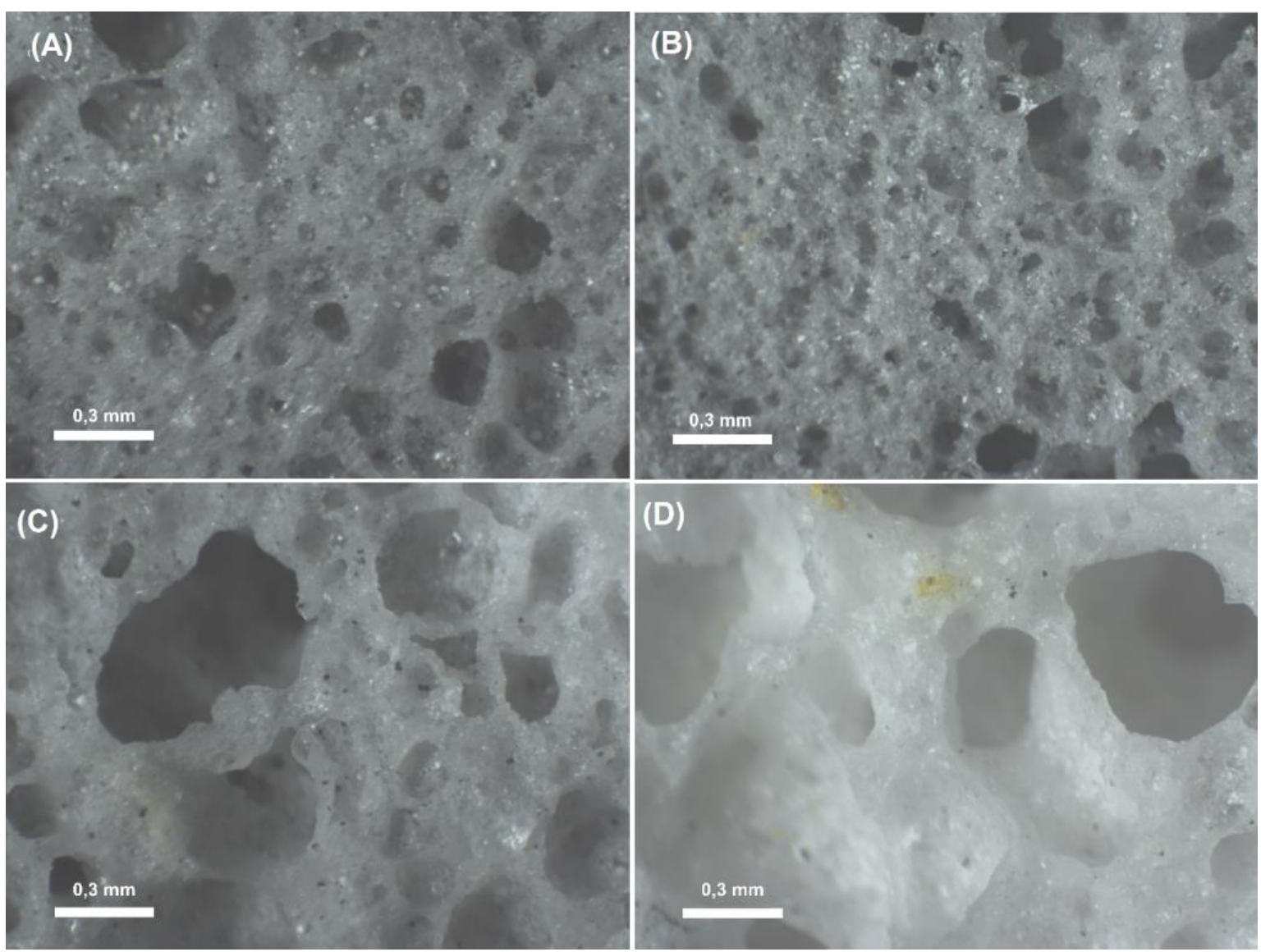

Figura 6: Micrografias das EVs obtidas nas temperaturas de (A) $700{ }^{\circ} \mathrm{C},(\mathrm{B}) 750{ }^{\circ} \mathrm{C}$, (C) $800{ }^{\circ} \mathrm{C}$ e (D) $850{ }^{\circ} \mathrm{C}$.

Tabela 3: Valores de diâmetro médio de poros $(\mathrm{mm})$ para espumas vítreas nas temperaturas de 700 a $850{ }^{\circ} \mathrm{C}$.

\begin{tabular}{c|c|c}
\hline $\begin{array}{c}\text { TEMPERATU- } \\
\text { RA }\left({ }^{\circ} \mathrm{C}\right)\end{array}$ & TAMANHO MÉDIO DE PORO & $\begin{array}{c}\text { DESVIO } \\
(\mathrm{mm})\end{array}$ \\
\hline 700 & 0,22 & PA- \\
\hline 750 & 0,42 & $\pm 0,18$ \\
\hline 800 & 0,70 & $\pm 0,52$ \\
\hline 850 & 2,39 & $\pm 0,56$ \\
\hline
\end{tabular}

Ao observar as amostras sintetizadas nas diferentes temperaturas (Fig. 6), percebe-se que a quantidade de poros diminutos aparentemente diminui com o aumento temperatura, o que é benéfico para a resistência mecânica [4], pois a redução de poros pequenos diminui a probabilidade de falhas críticas existentes. EVs obtidas à $700{ }^{\circ} \mathrm{C}$ apresentaram tamanho médios de poros de $0,22 \mathrm{~mm}$, relativamente baixo quando comparado aos demais. Para CPs, obtidos à $750{ }^{\circ} \mathrm{C}$, o tamanho médio dos poros foi de $0,42 \mathrm{~mm}$, esse valor é $91 \%$ superior àquele encontrado para queima de $700{ }^{\circ} \mathrm{C}$. Os CPs queimados nessas temperaturas apresentaram estrutura fechada e porosidade não homogênea.

As micrografias das EVs de 800 e $850^{\circ} \mathrm{C}$ (Figs. 6(C) e (D)) apresentam paredes espessas e com distribuição dupla, ou seja, há a presença de poros menores circundando os poros maiores. Essa distribuição dupla pode induzir fenômenos de coalescência ou crescimento de poros [26]. Esse fenômeno ocorre devido a dimi- 
nuição da viscosidade da matriz vítrea com o aumento da temperatura de queima. Quanto menor a viscosidade do vidro, menor é a sua resistência à pressão de $\mathrm{CO}_{2}$ (formado pela decomposição do AE). Essa baixa resistência acarreta numa maior liberação desse gás na massa cerâmica, fazendo com que as paredes dos poros se rompam, liberando o $\mathrm{CO}_{2}$ do seu interior. $\mathrm{Se}$ o $\mathrm{CO}_{2}$ alcançar a superfície do corpo cerâmico, ocorre a sua liberação e consequente encolhimento volumétrico da peça, além de formar poros maiores e mais heterogêneos, além de porosidade aberta. Esses resultados estão de acordo com aqueles obtidos por Méar et al. para temperaturas acima de $800{ }^{\circ} \mathrm{C}[27]$.

\section{CONCLUSÕES}

Foi possível obter espumas vítreas com baixo impacto ambiental e com características comerciais utilizado como matéria-prima o resíduo de vidro de lâmpada fluorescente e resíduo de casca de ovo vermelho como agente espumante. Verificou-se que a formação da espuma vítrea é diretamente dependente da temperatura de queima, assim como da temperatura de decomposição do agente espumante. Os melhores resultados foram obtidos a $750{ }^{\circ} \mathrm{C}$, na qual se obteve espumas vítreas com expansão de $266 \%$ e densidade de $0,37 \mathrm{~g} / \mathrm{cm}^{3}$, valor esse muito próximo da faixa de densidade de produtos comerciais. Através da análise microestrutural é possível verificar que EVs obtidas a baixas temperaturas, 700 e $750{ }^{\circ} \mathrm{C}$, apresentam porosidade não homogênea e fechada. Vale ressaltar que o presente estudo apresenta uma alternativa para destinação final de resíduos sólidos e consequente aplicação de valor comercial para rejeitos de lâmpadas fluorescentes e cascas de ovos. Além disso, o material sintetizado é inovador, sustentável e de baixo custo, que pode desempenhar funções de isolamento acústico e térmico.

\section{AGRADECIMENTOS}

Os autores agradecem a Coordenação de Aperfeiçoamento de Pessoal de Nível Superior - CAPES, a empresa Recilux e ao Laboratório de Materiais Cerâmicos - LACER da Universidade Federal do Rio Grande do Sul/Porto Alegre - UFRGS.

\section{BIBLIOGRAFIA}

[1] BERNARDO, E., SCARINCI, G., HREGLICH, S., "Effect of time and furnace atmosphere on the sintering of glasses from dismantled cathode ray tubes", Journal of the European Ceramic Society, v. 27, n.2-3, pp. 1637-1643, Jun 2007.

[2] PASCAL, G.Y, FRANÇOIS, O. M., "Characterization of lead, barium and strontium leachability from foam glasses elaborated using waste cathode ray-tube glasses", Journal of Hazardous Materials, v.185, n.1, pp. 236-241, Jan 2011.

[3] GUO, Y., ZHANG, Y., HUANG, H., et al., "Novel glass ceramic foams materials based on polishing porcelain waste using the carbon ash waste as foaming agent", Construction and Building Materials, v. 125, pp. 1093-1100, Oct 2016.

[4] ZHU, M., JI, R., LI, Z., et al., "Preparation of glass ceramic foams for thermal insulation applications from coal fly ash and waste glass", Construction and Building Materials, v. 112, pp. 398 - 405, Jun 2016.

[5] FERNANDES, H.R, ANDREOLA, F., BARBIERI, L., et al., "The use of eggshells to produce Cathode Ray Tube (CRT) glass foams”, Ceramics International, v. 39, n.8, pp. 9071-9078, Dec 2013.

[6] VIERERBL, L., VINŠ, M., LAHODOVÁ, Z., et al., "Mercury mass measurement in fluorescent lamps via neutron activation analysis", Radiation Physics and Chemistry, v. 116, pp.56-59, Nov 2015.

[7] TAN, Q., LI, J. “A study of waste fluorescent lamp generation in mainland China”, Journal of Cleaner Production, v. 81, pp.227-233, Oct 2014.

[8] SCHEUHAMMER, A., BRAUNE B., CHAN H. M., et al., "Recent progress on our understanding of the biological effects of mercury in fish and wildlife in the Canadian Arctic", Science of The Total Environment, v. 509-510, pp.91-103, Mar 2015.

[9] WILDEMANN, T. M., WEBER, L. P., SICILIANO, S. D. "Combined exposure to lead, inorganic mercury and methylmercury shows deviation from additivity for cardiovascular toxicity in rats", Journal of Applied Toxicology, v. 35, n.8, pp. 918-926, Aug 2015.

[10] MUGONI, C., MONTORSI, M., SILIGARDI C., et al., "Design of glass foams with low environmental impact”, Ceramics International, v. 41, n. 3, pp. 3400-3408, Apr 2015. 
[11] POKORNY, A., VICENZI, J., BERGMANN, C.P. "Influence of Heating Rate on the Microstructure of Glass Foams", Waste Management \& Research (ISWA), v. 1, pp. 1-8, Apr 2010.

[12] KÖNIG, J, PETERSEN, R.R, YUE, Y. "Fabrication of highly insulating foam glass made from CRT panel", Ceramics International, v. 41, n. 8, pp.9 793-9800, Sep 2015.

[13] ZHANG, Q., HE, F., SHU, H., et al., "Preparation of high strength glass ceramic foams from waste cathode ray tube and germanium tailings", Construction and Building Materials, v. 111, pp. 105-110, May 2016.

[14] RINCÓN, A., GIACOMELLO, G., PASETTO, M., et al., "Novel 'inorganic gel casting' process for the manufacturing of glass foams", Journal of the European Ceramic Society, v. 37 n. 5, pp. 2227-2234, May 2017.

[15] SPIRIDONOV, Y.A., ORLOVA L. A., "Problems of foam glass production", Glass and Ceramics, v. 9, pp. 313-314, 2003.

[16] ØSTERGAARD, M., PETERSEN, R., KÖNIG, J., et al., "Influence of foaming agents on solid thermal conductivity of foam glasses prepared from CRT panel glass", Journal of Non-Crystalline Solids, pp. 1-6, Apr. 2017.

[17] MITTAL, A., TEOTIA, M., SONI, R.K., et al.,"Applications of egg shell and egg shell membrane as adsorbents: A review”, Journal of Molecular Liquids, v. 223, pp.376-387, Nov 2016.

[18] VIEIRA, C. M. F., MORAIS, A. S. C., MONTEIRO, S. N., et al., "Teste industrial de cerâmica vermelha incorporada com resíduo de vidro de lâmpada fluorescente", Cerâmica, v. 62, n. 364, p.376-385, Dez. 2016.

[19] HINCKE, M. T., NYS, Y., GAUTRON, J., et al., "The eggshell: structure, composition and mineralization”, Frontiers in Bioscience, v.17, pp.1266-1280, Jan 2012.

[20] RENDER, D., SAMUEL, T., KING, H., et al., "Biomaterial-Derived Calcium Carbonate Nanoparticles for Enteric Drug Delivery”, Journal of Nanomaterials, v. 2016, pp. 1-8, Mar 2016.

[21] ATTILA, Y., GUDEN, M., TAŞDEMIRCI, A. "Foam glass processing using a polishing glass powder residue", Ceramics International. v. 39, n. 5, pp. 5869-5877, Jul 2013.

[22] SCHEFFLER, M., COLOMBO, P., Cellular Ceramics: Structure, Manufacturing, Properties and Applications, Weinheim, Wiley-VCH Verlag GmbH \& Co, 2005.

[23] KÖNIG, J., PETERSEN, R. R., YUE, Y. "Influence of the glass particle size on the foaming process and physical characteristics of foam glasses", Journal of Non-crystalline Solids, v. 447, pp.190-197, Sep 2016.

[24] MOSADDEGH, E., HASSANKHANI, A., "Preparation and characterization of nano-CaO based on eggshell waste: Novel and green catalytic approach to highly efficient synthesis of pyrano[4,3-b]pyrans", Chinese Journal of Catalysis, v. 35, n. 3, pp. 351-356, Mar 2014.

[25] KÖNIG, J., PETERSEN, R. R., YUE, Y. "Influence of the glass-calcium carbonate mixture's characteristics on the foaming process and the properties of the foam glass", Journal of The European Ceramic Society. pp. 1591-1598. Jun 2014.

[26] FERNANDES, H. R., FERREIRA, D., ANDREOLA, F., et al.,. "Environmental friendly management of CRT glass by foaming with waste egg shells, calcite or dolomite", Ceramics International, v. 40, pp. 13371-13379, Sep 2014

[27] MÉAR, F., YOT, P., VIENNOIS, R., et al., "Mechanical behaviour and thermal and electrical properties of foam glass", Ceramics International, v. 33, n. 4, pp. 543-550, May 2007. 